\title{
Studying Genetic Algorithm and Particle Swarm Optimization In Machine Learning
}

\author{
Shameen Basharat ${ }^{1}$, Sapna Jain ${ }^{2}$ \\ \{shameenbasharat@yahoo.com,drsapnajain@jamiahamdard.ac.in\}
}

Department of CSE, SEST, Jamia Hamdard, New Delhi 110062

\begin{abstract}
Each streamlining issue can be disentangled into the basic minimum-seeking dilemma. Which way is the most limited in separation? Which choice is the least expensive? Such inquiries are surely key to the investigation of optimisation. The accomplishment of genetic algorithm and swarm intelligence in managing enhancement issues is their common capacity to progressively find minima through straightforward, nearby associations of potential arrangements. This paper learns about streamlining methods like Genetic Algorithm utilizing Swarm Intelligence.
\end{abstract}

Keywords: Genetic algorithm, Swarm intelligence, Machine learning, Fitness function

\section{Introduction}

$\mathrm{AI}$ is an innovation that permits PCs to gain legitimately from models and experience form data. There are hard coded rules which clarify how an issue is to be handled bit by bit. On the contrary, AI frameworks are set an undertaking, and given a lot of information to use as instances of how this assignment can be accomplished or from which to distinguish designs. It very well may be thought of as narrow AI: AI underpins intelligent frameworks, which can become familiar with a specific capacity, given an arrangement of information to learn from. Machine learning permits PC frameworks to gain straightforwardly from models, information, and experience. Through empowering PCs to perform specific errands cleverly, as of late there have been many energizing revelations right now has made it progressively supportive to utilize.

Inside the field itself there have likewise been algorithmic advances, which have given AI more noteworthy force. Because of these advances, frameworks which just a couple of years prior performed at discernibly underneath human levels would now be able to beat people at some particular assignments. Numerous individuals presently interface with frameworks dependent on AI consistently, for instance in picture acknowledgment frameworks, for example, those utilized via web-based networking media. As the field grows further, AI shows guarantee of supporting possibly transformative advances in a scope of territories, and the social and monetary open doors which follow are noteworthy.

\section{Background}

AI manages the plan of calculations that license PCs to create practices dependent on 
experimental information. In AI the PC learns. A student can exploit guides to imprison qualities of significance of their unfamiliar basic probability distribution. The models delineate the connection between the info and wanted outcome. A significant assignment of AI explore is to naturally figure out how to recognize multifaceted examples and settle on insightful choices dependent on models. The arrangement of every conceivable conduct given every conceivable info is too huge to be in any way secured by the arrangement of watched models. A classifier framework is a framework that adapts linguistically straightforward standards called classifiers through credit task and rule disclosure systems. These frameworks perceive new data consistently from the earth. They develop assumptions without altering the acquired capabilities.

The above can likewise be utilized to make a specialist framework a reality. Classifier frameworks decide the positioning among the populace individuals by means of numerous communications with nature whereby the quality changes happen through the division of credit subsystem of classifier framework.

The parts of a classifier framework are

A. Information interface - It utilizes finders and changes over the twofold structure into standard messages.

B. Classifiers - Classifiers process messages utilizing framework techniques.

C. Message list - Message list has the rundown of current messages

D. Yield interface - It creates the yield in the ideal structure.

Consequently, the student must take a wide view from the given models, in order to have the option to manufacture a valuable yield in new cases.

\section{Genetic algorithm}

A hereditary calculation emulates the procedure of common advancement used to produce valuable answers for enhancement and search issues. Hereditary calculations are a subset of transformative calculations which tackles advancement issues utilizing procedures propelled by common development, for example, legacy, change, determination, and hybrid. Holland strategy thinks about the job of change and furthermore uses hereditary recombination that is hybrid to locate the ideal arrangement.

Five phases are considered in a genetic algorithm.

1. Initial population

2. Fitness function

3. Selection

4. Crossover

5. Mutation 
Initial population: The procedure starts with a lot of people which is known as a Population. Every individual is an answer for the issue you need to settle. An individual is portrayed by a lot of parameters (factors) known as Genes. Qualities are joined into a string to shape a Chromosome (solution).In a hereditary calculation, the arrangement of qualities of an individual is spoken to utilizing a string, as far as a letters in order as in figure1.

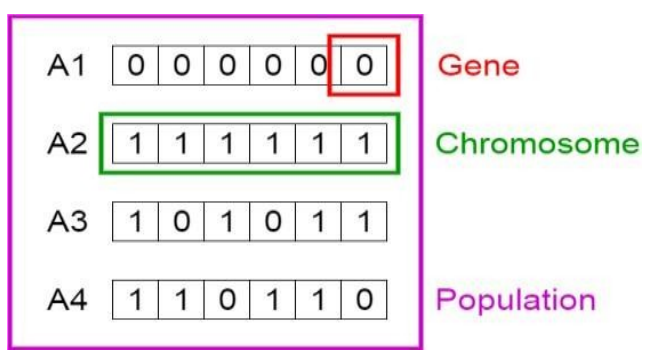

Figure1 Initial Population

Fitness function: The wellness work decides how fit an individual is (the capacity of a person to contend with others). It gives a wellness score to every person. The likelihood that an individual will be chosen for generation depends on its wellness score.

Selection: The thought of choice stage is to choose the fittest people and let them pass their qualities to the people to come. Two sets of people (guardians) are chosen dependent on their wellness scores. People with high wellness have increasingly opportunity to be chosen for generation.

Crossover: Crossover is the most critical stage in a hereditary calculation. For each pair of guardians to be mated, a hybrid point is picked aimlessly from inside the qualities. For instance, consider the hybrid point tobe 3 as appeared in figure 1 beneath. 


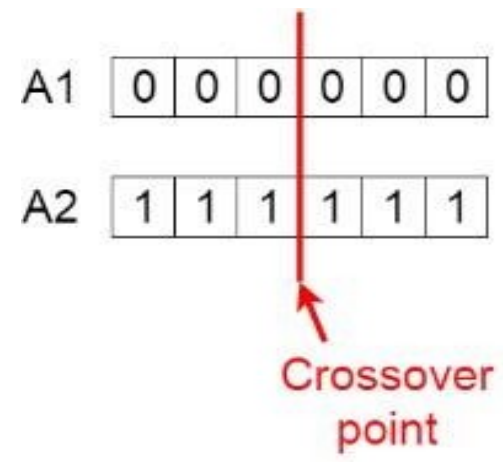

Figure 2 Crossover

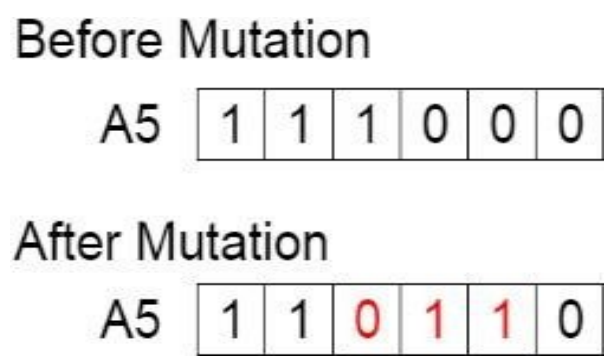

Figure3 Mutation

3.1 Implementation and result analysis 


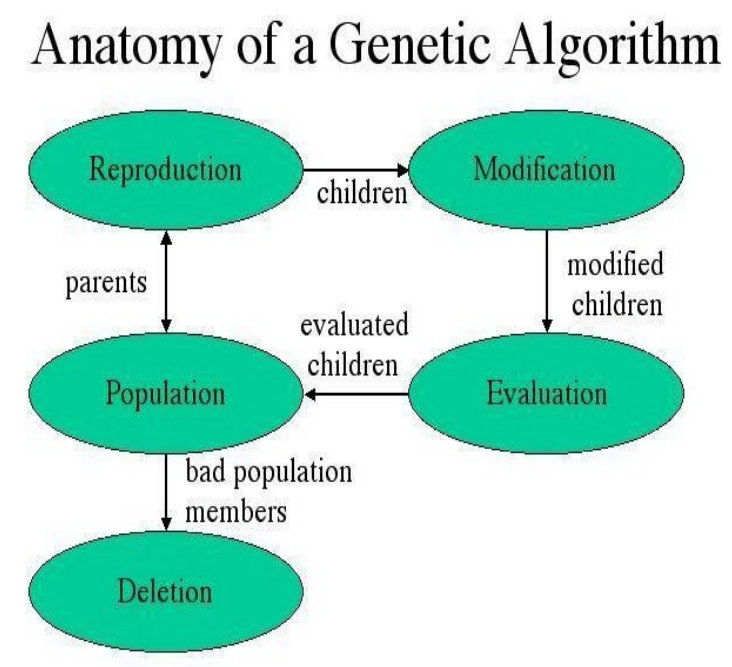

Fig 4. Anatomy of Genetic Algorithm

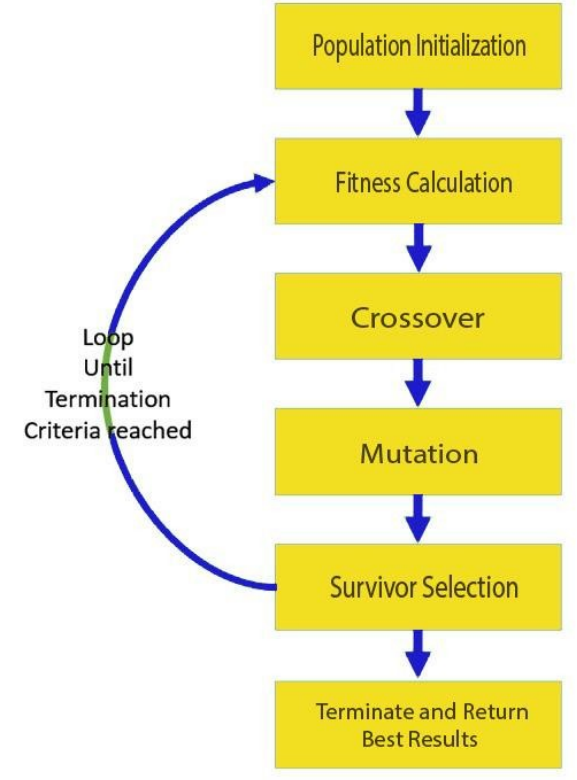

Fig 5. Working Process of GA

The working procedure of hereditary calculation, the means to understand a fundamental GA are recorded:

Stage 1: Represent the issue variable area as a chromosome of fixed length; pick 
the size of the chromosome populace N, the hybrid likelihood Pc and the transformation likelihood Pm

Stage 2: Define a wellness capacity to quantify the exhibition of an individual

chromosome in the issue space. Stage 3: Randomly produce an underlying populace of

size $\mathrm{N}: \mathrm{N} \mathrm{x}, \mathrm{x}, \ldots, \mathrm{x} 12$

Stage 4: Calculate the wellness of every individual chromosome.

Stage 5: Select a couple of chromosomes for mating from the present populace

Stage 6: Create a couple of posterity chromosomes by applying

the hereditary administrators. Stage 7: Place the made posterity

chromosomes in the new populace.

Stage 8: Repeat Step 5 until the size of the new populace rises to that

of beginning populace. Stage 9: Replace the underlying (parent)

chromosome populace with the new (posterity) populace.

A GA is an iterative procedure. Every cycle is known as a generation. Usually number of generations for a basic GA can go from 50 to more than 500. A typical practice is to end a GA after a predefined number of ages and afterward inspect the best chromosomes in the populace. On the off chance that no palatable arrangement is discovered, at that point the $\mathrm{GA}$ is restarted.

\subsection{Applications}

1. Economics - GAs are likewise used to portray different monetary models like the spider web model, game hypothesis harmony goals, resource valuing, and so forth.

2. Neural Networks - GAs are likewise used to prepare neural systems, especially repetitive neural systems.

3. Parallelization - GAs additionally have generally excellent equal abilities, and end up being compelling methods in taking care of specific issues, and furthermore give a decent region to explore.

4. Image Processing - GAs are utilized for different computerized picture preparing (DIP) assignments also like thick pixel coordinating. 
5. Vehicle steering issues - With different delicate time windows, various stations and a heterogeneous armada.

6. Scheduling applications - GAs are utilized to take care of different booking issues also, especially the time postponing issue.

7. Machine Learning - as of now examined, hereditary qualities based AI (GBML) is a specialty region in $\mathrm{AI}$.

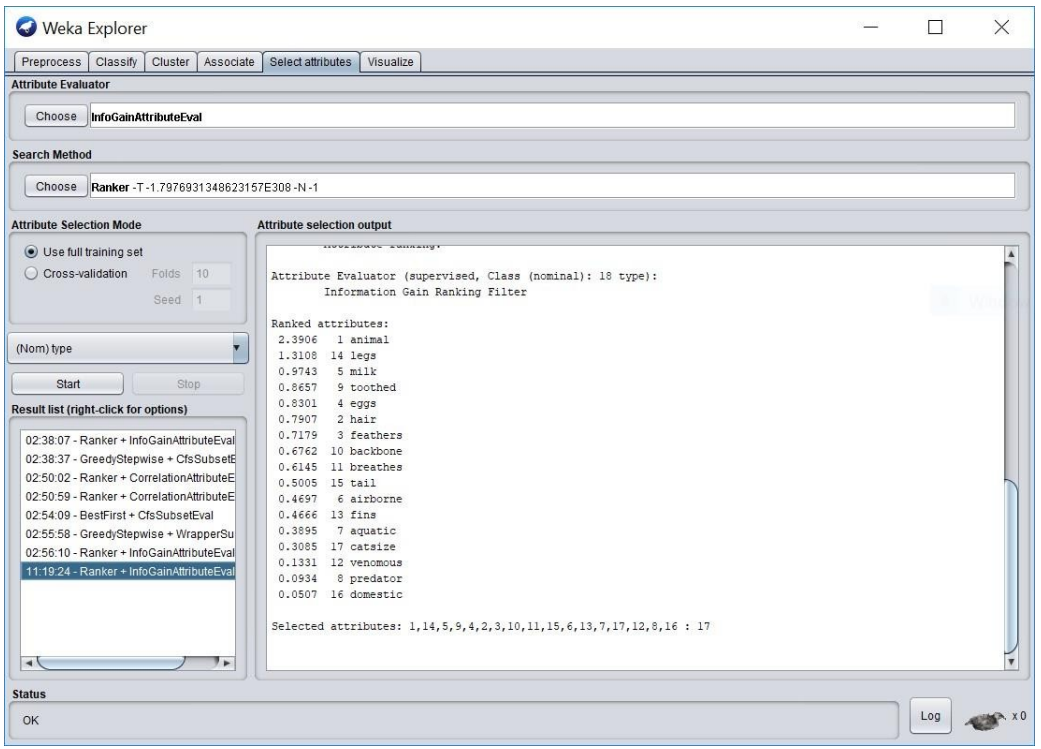

Fig 6. Data of Animals in zoo 


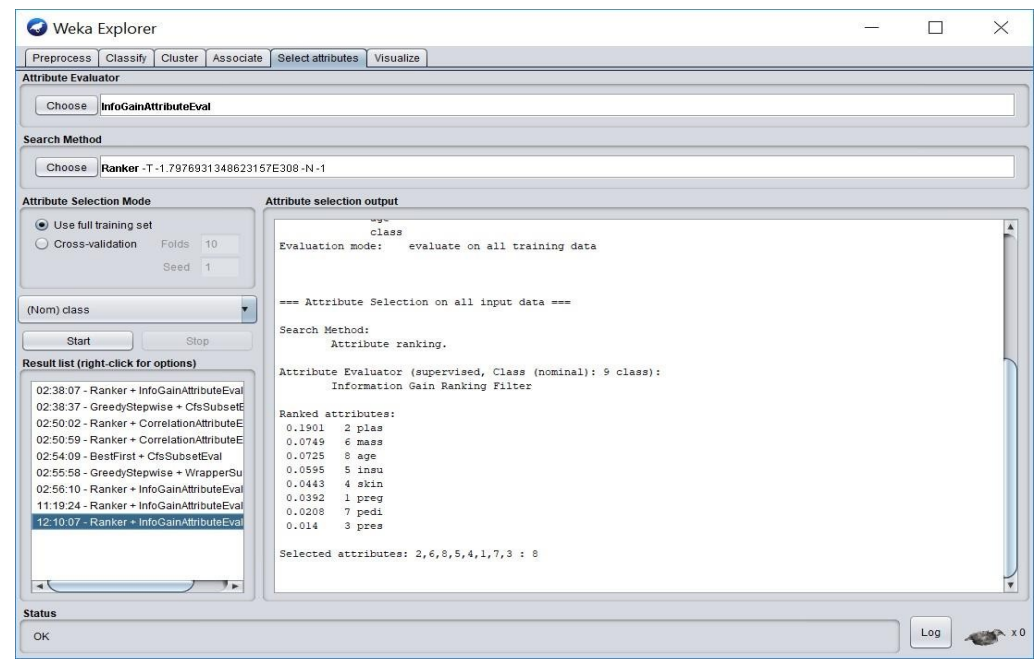

Fig 7. Data of Diabetes patients

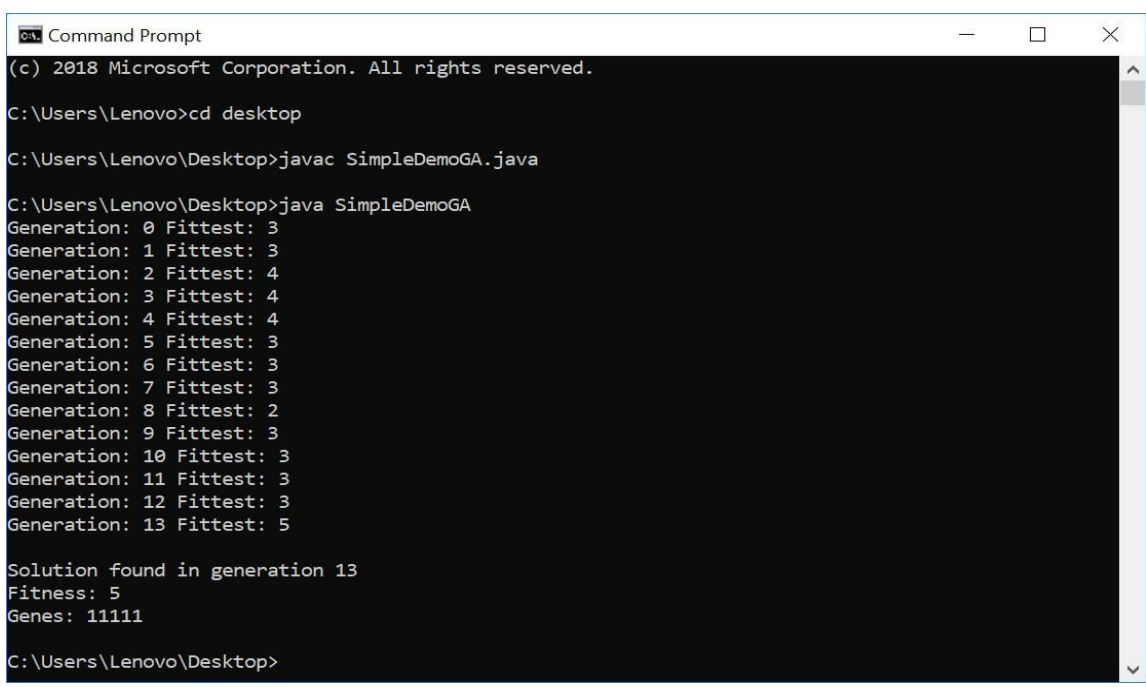

Fig 8. Java code output implementing genetic algorithm 


\subsection{Advantages}

- Optimization issues are tackled with numerous arrangements

- Can take care of issues with non-parametrical issues, multi-dimensional, non-persistent and non-differential

- Optimization issue that can be clarified with chromosome encoding can be tackled utilizing GA

- Large number of arrangement set can be immediately filtered

\subsection{Disadvantages}

- No assurance that GA will consistently give a worldwide ideal

- Cannot guarantee that GA will give consistent advancement reaction time

- Applications of GA continuously framework controls are restricted because of assembly and irregular arrangements

\section{Particle swarm optimization}

Molecule swarm improvement (PSO) is a population based technique that motivated from the conduct (data trade) of the feathered creatures in a swarm. In PSO the populace is known as a swarm and the people are called particles. In the inquiry space, every molecule moves with a speed. The molecule adjusts this speed because of the data trade among it and other neighbours. At every emphasis, the molecule utilizes a memory so as to spare its best position and the general best molecule positions as appeared in figure 9. The best molecule position is spared as a best nearby position, which was doled out to a local molecule, while the general best molecule position is spared as a best worldwide position, which was appointed to all particles in the swarm. The calculation monitors three worldwide factors:

- Target worth or condition

- Global best (gBest) esteem

- Stopping esteem

Every molecule comprises of Data speaking to an answer, A Velocity esteem and An individual best (pBest) esteem. PSO and GA are a lot of comparable in their innate equal qualities, while tests show that they have their particular points of interest when taking care of various issues. What we might want to do is to acquire both their magnificent highlights by incorporating the two calculations. In the proposed approach, the calculation instated with a populace of arbitrary arrangements and looks for optima by going into the hunt space. During this movement an advancement of this arrangement is performed by coordinating PSO and GA. 


\section{Conclusion}

Hereditary Algorithm and Swarm Intelligence are the two insightful strategies presently utilizing as a heuristic strategy for taking care of complex issues (like NP-Hard) that are elusive arrangements utilizing ordinary existing procedure. Each calculation talked about has benefits and faults. They worked with various structures in various situations. These calculations don't ensure that it will consistently give ideal arrangement. So as to upgrade the ability of tackling issues and improve their presentation, cross breed calculations are being created by consolidating these calculations among one another. Their applications are still in investigation. A portion of their notable applications are organize the executives, mechanical autonomy, neural systems, AI, traffic control and so on. Among these calculations, PSO is the most up to date and research on it is toward the starting stage. PSO has no any systematically figuring strategy or any clear scientific establishment yet at the same time generally utilized in different territory utilizing its straightforward computation procedure. PSO is as yet frail in hypothetical establishment so the greater part of its ideal arrangement can't ensure in principle. Further research in PSO's hypothetical establishment ought to be done so as to get it flawlessness. Its application regions ought to be investigated further. GA works just in stable condition with discrete factors. It ought to be able to work in powerful condition with nonstop factor and it should ensure union so further improvement is required. Worldwide pursuit method ought to likewise be actualized. In ACO beginning and goal hub is characterized before. ACO's concern zones ought to be broadened further by making it able to take care of issues that don't characterize its size, shape and multifaceted nature ahead of time.

\section{References}

[1]. A Comparison of Particle Swarm Optimization and the Genetic Algorithm -

$\underline{\text { Rania Hassan Babak Cohanim ,Olivier de Weck and Gerhard Venter }}$

[2] A hybrid particle swarm optimization and genetic algorithm with population partitioning for large

Ahmed F.Ali ${ }^{\mathrm{ab}}$ Mohamed A.Tawhid ${ }^{\mathrm{ac}}$

[3] "Routing multiple description video over NLOS small cell backhaul", Bessie Malila, Olabisi Falowo,

Neco Ventura. 2017 IEEE AFRICON, 2017 scale optimization problems

[4] Integrating particle swarm optimization with genetic algorithms for solving nonlinear optimization problems - Ahamed Al Malki, Mohamed M. Rizk, M. A. El-

Shorbagy, A. A. Mousa

[5] A Sequential Hybridization of Genetic Algorithm and Particle Swarm Optimization for the Optimal Reactive Power Flow Imene Cherki, Abdelkader Chaker, Zohra Djidar, Naima Khalfallah and Fadela Benzergua 
[6] Particle Swarm Optimization and Differential Evolution Algorithms: Technical Analysis, Applications and Hybridization Perspectives - Swagatam Das, Ajith Abraham, and Amit Konar

[7] A Swarm Optimization Genetic Algorithm Based on Quantum-Behaved Particle Swarm Optimization -

$\underline{\text { Tao Sun and Ming-hai Xu }}$

[8] Particle Swarm Optimization: A survey of historical and recent developments with hybridization perspectives - Saptarshi Sengupta *, Sanchita Basak and Richard Alan Peters II

[9] A comparative study of particle swarm optimization and genetic algorithm- Saman M. Almufti, Amar Yahya Zebari, Herman Khalid Omer

[10] A Comparative Study of Genetic Algorithm and the Particle Swarm OptimizationShahid Shabir, Dr. Ruchi Singla. 